\title{
Age aspects of habitability
}

\author{
M. Safonova', J. Murthy' and Yu. A. Shchekinov ${ }^{2}$ \\ ${ }^{1}$ Indian Institute of Astrophysics, Bangalore, India e-mail: rita@iiap.res.in \\ ${ }^{2}$ Department of Space Physics, SFU, Rostov on Don, Russia
}

\begin{abstract}
A 'habitable zone' of a star is defined as a range of orbits within which a rocky planet can support liquid water on its surface. The most intriguing question driving the search for habitable planets is whether they host life. But is the age of the planet important for its habitability? If we define habitability as the ability of a planet to beget life, then probably it is not. After all, life on Earth has developed within only $\sim 800 \mathrm{Myr}$ after its formation - the carbon isotope change detected in the oldest rocks indicates the existence of already active life at least $3.8 \mathrm{Gyr}$ ago. If, however, we define habitability as our ability to detect life on the surface of exoplanets, then age becomes a crucial parameter. Only after life had evolved sufficiently complex to change its environment on a planetary scale, can we detect it remotely through its imprint on the atmosphere - the so-called biosignatures, out of which the photosynthetic oxygen is the most prominent indicator of developed (complex) life as we know it. Thus, photosynthesis is a powerful biogenic engine that is known to have changed our planet's global atmospheric properties. The importance of planetary age for the detectability of life as we know it follows from the fact that this primary process, photosynthesis, is endothermic with an activation energy higher than temperatures in habitable zones, and is sensitive to the particular thermal conditions of the planet. Therefore, the onset of photosynthesis on planets in habitable zones may take much longer time than the planetary age. The knowledge of the age of a planet is necessary for developing a strategy to search for exoplanets carrying complex (developed) life - many confirmed potentially habitable planets are too young (orbiting Population I stars) and may not have had enough time to develop and/or sustain detectable life. In the last decade, many planets orbiting old (9-13 Gyr) metal-poor Population II stars have been discovered. Such planets had had enough time to develop necessary chains of chemical reactions and may carry detectable life if located in a habitable zone. These old planets should be primary targets in search for the extraterrestrial life.

Received 3 May 2015, accepted 9 June 2015, first published online 11 August 2015
\end{abstract}

Key words: formation, habitability, photosynthesis, planetary systems.

\section{Introduction}

Habitability may be quantitatively defined as a measure of the ability of a planet to develop and sustain life (Schulze-Makuch et al. 2011); its maximum is set as 1 for a planet where life as we know it has formed, thus it is 1 for the Earth. The requirement for a planet to be called habitable (or potentially habitable) ${ }^{1}$ is that the planet is located within the host's habitable zone (HZ) and has terrestrial characteristics: rocky, with a mass range of 0.1-10 Earth masses and a radius range of $0.5-2$ Earth radii ${ }^{2}$. A $\mathrm{HZ}$ is conservatively defined as a region where a planet can support liquid water on the surface (Huang 1959). The concept of an $\mathrm{HZ}$ is, however, a constantly evolving one, and many different variations of it have been since suggested (see, e.g., an excellent review by Lammer et al. (2009) and references therein

1 Both definitions habitable and potentially habitable are used in the literature, meaning essentially the same, but see Sec. 4 for our discussion on the definition.

2 The latest simulations have shown that after $\sim 1.7$ Earth radii the planets are of increasingly lower density, indicating that they are less rocky and more like mini-Neptunes, placing the Earth's twin limit on the radius (for ex. Buchhave et al. 2014), though uncertainties remain, see, e.g. Torres et al. (2015). and Heller \& Armstrong (2014) as a more recent one). Biogenic elements (such as $\mathrm{C}, \mathrm{H}, \mathrm{N}, \mathrm{O}, \mathrm{P}$ and $\mathrm{S}$ ) have also been considered as necessary complementary factors for habitability (Chyba \& Hand 2005), but their presence is implied by the existence of water as they are produced in the same stars (Heger \& Woosley 2002; Umeda \& Nomoto 2005).

We would like to stress here that throughout the paper, when we talk about detecting life on exoplanets, we still mean life as we know it, the presence of which we are able to establish through predictable changes in planetary atmospheres. Even on Earth, there is a possibility of a different kind of life not based on a usual triad - DNA-protein-lipid; see, for example, the discussion on a 'shadow' biosphere by Davies et al. (2009). But just as on Earth we are not able to find it (yet) as we do not know 'where or what to look for', we may not be able to distinguish these different kinds of life from the natural environments of exoplanets. Hence, when we talk about biosignatures, we mean only biosignatures that our kind of life produces - oxygen, ozone, nitrous oxide, etc. (e.g., Seager et al. 2012). A planet may host life as we know it (in other words, be not just habitable but inhabited), but we will still not detect it unless it has evolved sufficiently to change its environment on a planetary scale, for instance, through the production of an oxygen atmosphere by 
photosynthetic organisms. Photosynthesis is currently the only geologically documented biogenic process (see, e.g., Lyons \& Reinhard, 2011; Fomina \& Biel 2014 and references therein) that can provide sufficient energy to modify the global planetary (or atmospheric) properties. The large free energy release per electron transfer and stability of the oxygen molecule due to its strong bonding ensures that an oxygen-rich atmosphere provides the largest feasible energy source for complex life (e.g., Catling et al. 2005). Therefore, by analogy with the Earth, we presume the presence of an oxygen atmosphere as necessary for a planet to host a complex life. Such life would have modified the global planetary (or atmospheric) properties to be noticed from space, and from very far away; after all, the closest potentially habitable planet (PHP) is at about 12 light years ( $\tau$ Ceti) and we cannot go there to verify. Even Mars might still be inhabited by a primitive subsurface biota which is undetectable without a local and detailed examination. It may also be possible for life to evolve in a manner that we have not anticipated, which, even if it changes the environment globally, would not be detectable simply because we are not looking for those particular changes. For example, aphotic life can exist in the subsurface oceans of Europa or Enceladus, but such life would be currently impossible for us to detect ex situ.

Biological methanogenesis was suggested as a rival to the photosynthesis process in changing the global environment and capable of enriching the exo-atmospheres with biogenic methane (Schindler \& Kasting 2000; Kharecha et al. 2005). Kharecha et al. (2005) has shown that the rate of biogenic methanogenesis in the atmosphere of an Archaen Earth could have been high enough to enrich the atmosphere with high concentration of biogenic methane. However, planets with reduced mantles might enrich their atmospheres by methane abiotically (e.g., Etiope \& Lollar 2013), and thus methane alone cannot guarantee habitability. From this point of view, methanogenic products are a less certain biosignature of Earth-like life than oxygen (Seager et al. 2012). Accounting for a competitive interrelation between metabolic and abiotic origins of methane, a more conservative understanding suggests that only the simultaneous presence of methane along with other biogases is a reliable indication of life (e.g., Selsis et al. 2002; Kaltenegger et al. 2007; Kiang et al. 2007; Kasting et al. 2014). It could also be that the planet never develops oxygenic photosynthetic life. In such cases, other biomarkers have been suggested; for example, dimethyl disulphide and $\mathrm{CH}_{3} \mathrm{Cl}$ may be detected in infrared (IR) in the planetary atmospheres of low-ultraviolet (UV) output stars (Domagal-Goldman et al. 2011).

Carter (1983) has pointed out that the timescale for the evolution of intelligence on the Earth $(\sim 5 \mathrm{Gyr})$ is comparable with the main sequence lifetime of the Sun $(\sim 10 \mathrm{Gyr})$. Lin et al. (2014) suggested that intelligent life on expolanets can be detected through the pollution it inflicts on the atmosphere. However, intelligent life, once evolved, is no longer in need of a very precisely defined biosphere - we can already create our own biospheric habitats on planets that are lifeless in our definition of habitability, e.g. Moon or Mars, though we are technologically intelligent for only a $0.0000026 \%$ of the time life exists on Earth: 100 years out of 3.6 Gyr. Therefore, intelligent life may not be so easily detectable, especially if they have had a longer time to evolve. However, to answer the most important question of 'are we alone', we do not necessarily need to find intelligent life. Even detection of a primitive life will have a profound impact on our civilization. Therefore, we need to concentrate on the period in a planet's history when the emerged life had already influenced the atmosphere of the planet in a way that we can possibly recognize.

We discuss here the importance of the age of the planet in the evaluation of whether that $\mathrm{HZ}$ planet contains life and whether that life is detectable. We examine the plausibility of a discovery of a habitable planet with detectable biota among the close (within $600 \mathrm{pc}$ ) neighbours of the Sun. We argue that variations in their albedos, orbits, diameters and other crucial parameters make the formation of a significant oxygen atmosphere take longer that the current planetary age and thus, life can be detectable on only half of the confirmed PHPs with a known age.

\section{Initial stages of habitability}

Necessary conditions for the developing of life are thought to include rocky surface and liquid water; however, the aspects connected with the stages preceding the onset of biological era are usually left out of consideration. Planetary age as a necessary condition for life to emerge was first stressed by Huang (1959) and implicitly mentioned by Crick \& Orgel (1973) in their concept of a Directed Panspermia.

In order to understand the importance of planetary age for the evolution of a detectable biosphere we will consider, as an example, the development of cyanobacteria and the related atmospheric oxidation (Irwin et al. 2014). This process involves several endothermic reactions and requires sufficiently high temperatures to be activated. In general, the temperature dependence of the photosynthetic rate is rather complicated and conditionally sensitive, with the effective activation energy being of the order of tens of $\mathrm{kJ} \mathrm{mol}^{-1}$ (Hikosaka et al. 2006), much higher than the typical equilibrium temperature on habitable planets. Thus small variations in atmospheric and crust properties can considerably inhibit photosynthesis and increase the growth time of the mass of cyanobacteria. This conclusion may be illustrated through the consideration of the elementary process of carboxylation of RuBP (ribulose-1,5bisphosphate: $\left.\mathrm{C}_{5} \mathrm{H}_{12} \mathrm{C}_{11} \mathrm{P}_{2}\right)$ in the dark Benson-BasshamCalvin cycle of photosynthesis (Benson et al. 1950; Farquhar et al. 1980). These photosynthetic reactions, controlled by enzymes, are known to be very sensitive to ambient temperatures with an optimum rate at about $40^{\circ} \mathrm{C}$, and a practically zero rate outside the temperature range of $0^{\circ}<t<60^{\circ} \mathrm{C}$ (Toole \& Toole 1997). Amongst other fundamental factors RuBP carboxylation is probably the most relevant one, determining the optimal temperature of photosynthesis, and is characterized by the activation energy $V_{\mathrm{C}} \simeq 30-60 \mathrm{~kJ} \mathrm{~mol}^{-1}$ at the growth temperature (Hikosaka et al. 2006). We can roughly characterize the RuBP carboxylation by the Arrhenius law

$$
k_{\mathrm{C}}=A \mathrm{e}^{-V_{\mathrm{C}} / T},
$$


where $k_{\mathrm{C}}$ is the rate constant, $A$ is the prefactor, and $T$ is the absolute temperature. The characteristic time of RuBP carboxylation is $\tau_{\mathrm{C}} \propto k_{\mathrm{C}}^{-1} \propto \exp \left(V_{\mathrm{C}} / T\right)$. Since the RuBP carboxylation is one of the main processes optimizing photosynthetic reactions, $\tau_{\mathrm{C}}$ can roughly characterize the rate of photosynthesis on a planet.

The range of variation in $\tau_{\mathrm{C}}$ on a habitable planet due to the uncertainty in the equilibrium temperature $T_{\mathrm{e}}$ is

$$
\frac{|\delta \tau|}{\tau_{\mathrm{e}}}=\frac{V_{\mathrm{C}}}{T_{\mathrm{e}}} \frac{|\delta T|}{T_{\mathrm{e}}},
$$

with $\tau_{\mathrm{e}}$ being a characteristic time of photosynthesis at $T_{\mathrm{e}}$. The equilibrium temperature $T_{\mathrm{e}}$, in turn, is calculated using planetary parameters inferred from the observations,

$$
T_{\mathrm{e}}=\left[\frac{L(1-a)}{16 \pi \sigma \varepsilon r^{2}}\right]^{1 / 4},
$$

where the uncertainties in the parameters determine the uncertainty in its estimate,

$$
\frac{|\delta T|}{T_{\mathrm{e}}}=\frac{1}{4}\left(\frac{|\delta L|}{L}+\frac{|\delta a|}{1-a}+|\delta \varepsilon|+2 \frac{|\delta r|}{r}\right) .
$$

Here $L$ is the luminosity of the central star, $a$ and $\varepsilon$ the planet's albedo and emissivity, $r$ the orbital radius, and $\sigma$ the Stephan-Boltzmann constant. It is readily seen that the actual time of the onset of photosynthesis for a given habitable planet might differ significantly from the value calculated from largely uncertain parameters that were, in turn, derived from observables. Indeed, uncertainties in estimates of the equilibrium temperature $|\delta T| / T_{\mathrm{e}}$ are heavily amplified for habitable planets with $V_{\mathrm{C}} / T_{\mathrm{e}} \simeq 10-20$ for $V_{\mathrm{C}} \simeq 30-60 \mathrm{~kJ} \mathrm{~mol}^{-1}$ and $T_{\mathrm{e}} \sim 300 \mathrm{~K}$, such that even relatively low observational errors in deriving the parameters in equation (4), of 5\% each, might result in $50-100 \%$ error in the estimates of the overall photosynthesis rate. If one considers oxidation of the Earth atmosphere as a process tracing the developing photosynthesis, the characteristic time for the growth of biota on early Earth can be estimated as the oxidation time, $\tau_{\mathrm{O}_{2}} \sim 2$ Gyr (Kasting 1993; Wille et al. 2007; Fomina \& Biel 2014). Therefore, a 50\% error in $\tau_{\mathrm{C}}$ may delay the possible onset of biological evolution on a planet by $1 \mathrm{Gyr}$, i.e. biogenesis might not start earlier than 3 Gyr from the planetary formation. In general, however, the problem of the photosynthetic process is much more complex, depending on many factors determined by thermal and nonthermal processes on a planet (Shizgal \& Arkos 1996; Hikosaka et al. 2006), and might be even more sensitive to variations in physical conditions. Even on the early Earth, physical conditions could have been such as to preclude the onset of biogenesis over a long time (Sagan 1974; Maher \& Stevenson 1988; Solomatov 2000).

From this point of view, the planetary habitability index (PHI) recently proposed by Schulze-Makuch et al. (2011) in the form

$$
\mathrm{PHI}_{0}=(S \cdot E \cdot C \cdot L)^{1 / 4},
$$

can be generalized with explicit inclusion of the age of the planet $t$ as

$$
\operatorname{PHI}(t)=\mathrm{PHI}_{0} \prod_{i}\left(1-\mathrm{e}^{-t / t_{i}}\right) .
$$

In equation (5), $S$ defines a stable substrate, $E$ the necessary energy supply, $C$ the polymeric chemistry and $L$ the liquid medium; all the variables here are in general vectors, while the corresponding scalars represent the norms of these vectors. In equation (6), the index $i$ denotes a chemical chain relevant for further biochemical evolution, and $t_{i}$ is its characteristic time. It is obvious that the asymptotic behaviour - approaching the maximum habitability - is controlled by the slowest process with the longest $t_{i}$.

\section{Other factors delaying the onset of habitability}

Sagan (1974) was the first to stress that harmful endogenous and exogeneous processes in the early Earth could postpone emergence of life on it. Such processes could be important even in the very initial primitive episodes of biogenesis and delay the formation of biota for up to billions of years. It is known from the ${ }^{182} \mathrm{~W}$ isotope dating that the late heavy bombardment of Earth, Moon and Mars lasted till about $3.8 \mathrm{Ga}$ (Schoenberg et al. 2002; Moynier et al. 2009; Robbins \& Hynek 2012). The Martian primitive atmosphere is believed to have been lost through catastrophic impacts about $4 \mathrm{Ga}$ (e.g., Melosh \& Vickery 1989, Webster et al. 2013). Evidence of a heavy bombardment in other exoplanet systems exists: collision-induced hot dust was detected in several young planetary systems. Spectral signatures of warm water- and carbon-rich dust in the $\mathrm{HZ}$ of a young $\sim 1.4 \mathrm{Ga}$ MS star $\eta$ Corvi (Lisse et al. 2012), and of host dust in seven sun-like stars (Wyatt et al. 2007) indicate recent frequent catastrophic collisions between asteroids, planetesimals or even possible planets (Song et al. 2005). Out of these seven stars, five are young systems within their first Gyr of life.

It is also well-known that solar-type stars remain very active in the first billion years of their life, sustaining conditions that are hostile to the survival of the atmosphere and to the planetary habitability. G-type stars, within the first $100 \mathrm{Myr}$ of reaching ZAMS, produce continuous flares of extreme-UV (EUV) radiation up to 100 times more intense than the present Sun, and have much denser and faster stellar winds with an average wind density of up to 1000 times higher. Low-mass K- and M-type stars remain X-ray and EUV-active longer than solartype stars, where EUV emission can be up to 3-4 times and 10100 times, respectively, higher than G-type stars of the same age; and active M-type stars could keep stellar winds in the $\mathrm{HZ}$ that are at least 10 times stronger than that of present Sun (France et al. 2013).

In recent simulations by Schaefer and Sasselov (2015) of the development of oceans on super-Earths, it was shown that though these planets keep their oceans for longer than the Earth (up to $10 \mathrm{Gyr}$ ), it also takes longer for them to develop the surface ocean due to the delayed start of volcanic outgassing that returns water back to the surface from the mantle. For super-Earths 5 times the Earth's mass, that would take about a 
billion years longer. The oceans are believed to have established on Earth $750 \mathrm{Myr}$ after formation, therefore superEarths would have their surface water established at only 2 Gyr after formation. After all, the Great Oxygenation Event about $2.5 \mathrm{Ga}$ (Anbar et al. 2007) was most likely induced by oceanic cyanobacteria, which allowed life to emerge on land about 480 - 360 Ma (Myr ago) (Kenrick and Crane, 1997).

\section{Potentially habitable planets}

At the time of writing, more than 1900 exoplanets have been confirmed (Extrasolar Planets Encyclopaedia, June 2015) with another 4000 waiting for confirmation (NASA Exoplanet Archive). The majority of detected planets are in the vicinity of the Sun, and their hosts are mostly young Population I (Pop I) stars with ages of hundreds of Myr to a few Gyr. The age distribution of the host stars with measured ages is shown in Fig. 1. 58\% of the host stars have ages of 4.5 Gyr and less, and more than one-third ( $\sim 38 \%)$ are younger than 3 Gyr. Simple statistics shows the median age of $\sim 3.8$ Gyr.

The fact that more than one-third of the planetary systems, discovered by ongoing exoplanetary missions, are younger than $\sim 3$ Gyr is not surprising, because the continuous star formation (SF) in the Galactic disk supplies young stars, and the fraction of hosts younger than $3 \mathrm{Gyr}$ represents that very fraction of Pop I stars that would be born provided the SF rate is nearly constant during the whole period of the thin disk formation. Most of the current exoplanet missions suffer from an observational bias - they mostly detect systems that are younger than the age at which life is presumed to have appeared on the Earth $^{3}$.

Incidentally, Fig. 1 shows a deficit of stars with ages $t>6$ Gyr. Assuming that Pop I stars, i.e. the thin Galactic disc, have started forming at about $10 \mathrm{Ga}$ (Chen et al. 2003; Carraro et al. 2007), one might expect the presence of such old stars in our vicinity in the proportion corresponding to the SF history in the early Galaxy. The most conservative assumption implies a constant SF rate, in which case one should expect the number of planet-hosting stars with ages $t>6 \mathrm{Gyr}$ of about $40 \%$. It is, however, believed that the SF was more active in the early epochs (Bouwens et al. 2007), therefore, the fraction of hosts older than 6 Gyr should be correspondingly higher. The reason for the decline in the number of the hosts in this age range is unclear and might, in particular, indicate that planetary systems lose planets with age.

About 40 PHPs are currently documented ${ }^{4}$, though extrapolation of Kepler's data shows that in our Galaxy alone there could be as many as 40 billion PHPs (Petigura et al. 2013). In Table 1, we show the data for PHPs for which the host ages were available in the literature. The fraction of young

3 The earliest geological/fossilized evidence for the existence of biota on Earth dates to $\sim 3.8-3.5 \mathrm{Ga}$; see Brack et al. (2010) and references therein.

4 See, for example, the online Habitable Exoplanets Catalog (HEC), maintained by the Planetary Habitability Laboratory at the University of Puerto Rico, Arecibo, http://phl.upr.edu/projects/habitable-exoplanets-catalog, but not exclusively.

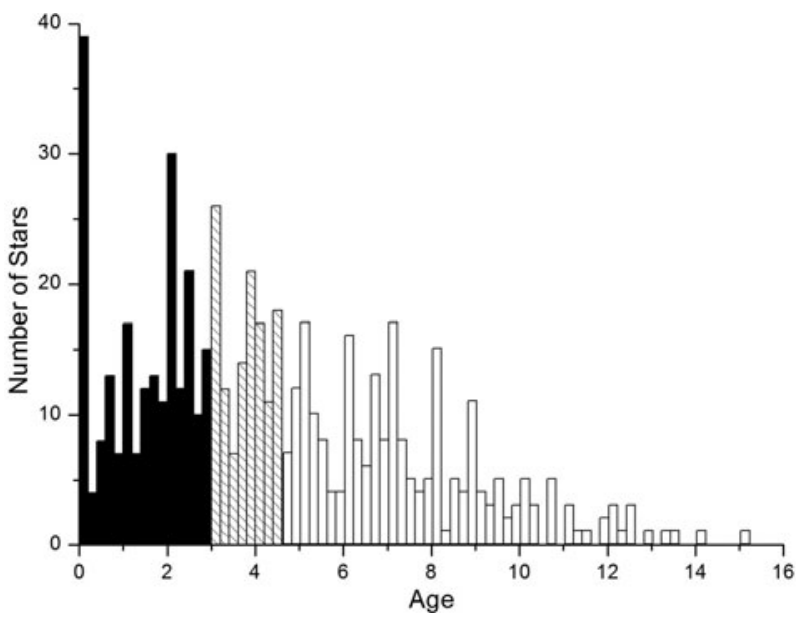

Fig. 1. Age distribution of the stars hosting confirmed planets (total 583 hosts with known ages at time of writing). We highlight the number of stars with ages below $3 \mathrm{Gyr}$ in black, and between 3 and 4.5 Gyr as hatched. The predominance of young host stars is clearly seen, which could be the effect of observational selection (Shchekinov et al. 2013). This figure was made using the Extrasolar Planets Encyclopaedia data.

planetary systems is nearly consistent with the age distribution of Pop I stars: among the 33 confirmed habitable planets with known ages more than half are $\lesssim 3.5$ Gyr old.

It seems reasonable to update the definitions in footnote 1 on page 1 as

1. $P H P$ - a rocky, terrestrial-size planet in an $\mathrm{HZ}$ of a star.

2. Habitable planet - a rocky, terrestrial-size planet in an $\mathrm{HZ}$ with detected surface water and some of the biogenic gases in atmosphere.

3. Inhabited planet - the best case scenario: a rocky, terrestrialsize planet in an HZ with simultaneous detection of species such as water, ozone, oxygen, nitrous oxide or methane in atmosphere, as proposed by e.g. Sagan et al. (1993) or Selsis et al. (2002).

We may expect only a primitive form of biota on the youngest planets ( $\lesssim 2$ Gyr) in Table 1, which would not be detectable. Biogenesis could have started, or even progressed to more advanced stages with an oxidized atmosphere, on older planets with ages from 2 to $4 \mathrm{Gyr}$. In the former case, one can expect that methane from metabolic reactions has already filled the atmosphere, while in the latter case, oxygen molecules at some level can appear in the atmosphere - though atmospheric oxygen on Earth appeared about $2.5 \mathrm{Ga}$, the Earth itself became visibly habitable only about 750-600 Ma, when the biosphere became active and complex enough to modify the environment to be noticed from space (e.g., Méndez et al. 2013). The traces of these gases may, in principle, be observed in sub-mm and micron wavelengths, provided the planets are orbiting low-mass stars $\left(0.5-0.8 M_{\odot}\right)$. Even if one-third of the low-mass stars in the sky host planets (Tutukov \& Fedorova 2012), there may be as many as a thousand planets within a $10 \mathrm{pc}$ vicinity with ages ranging from Myr to a few Gyr. 
Table 1. Host ages for confirmed PHPs

\begin{tabular}{|c|c|c|c|c|c|}
\hline Star & Planet(s) & Age estimate (Gyr) & Metallicity [Fe/H] & Distance $(\mathrm{pc})$ & Ref. to age \\
\hline Kepler 61 & Kepler-61 b $\mathrm{b}^{\mathrm{a}}$ & $\sim 1$ & 0.03 & 326 & 1 \\
\hline Gliese $667 \mathrm{C}$ & Gl $667 \mathrm{c}$ & $<2 ; 2-5 ;>2$ & -0.59 & 7.24 & $1 ; 2 ; 3$ \\
\hline Kepler 62 & Kepler-62 e, f & $7 \pm 4$ & -0.37 & 368 & 4 \\
\hline Kapteyn's & Kapteyn's b & $10-12$ & -0.99 & 3.91 & 5 \\
\hline Gliese 163 & Gl $163 \mathrm{c}$ & $3.0(+7 .,-2.) ;>2 ; 6 \pm 5$ & 0.1 & 15 & $1 ; 7 ; 8$ \\
\hline HD 40307 & HD $40307 \mathrm{~g}$ & $1.2 \pm 0.2 ; 4.5 ; 6.1$ & -0.31 & 12.8 & $9 ; 10$ \\
\hline HD 85512 & HD $85512 \mathrm{~b}$ & $5.61 \pm 0.61$ & -0.33 & 11 & 11 \\
\hline Kepler 22 & Kepler-22 $b^{a}$ & $\sim 4$ & -0.29 & 190 & 12 \\
\hline Gliese 832 & GJ $832 \mathrm{c}$ & 9.24 & $-0.31 \pm 0.2$ & 4.95 & 13 \\
\hline Kepler 186 & Kepler-186 f & $4 \pm 0.6$ & $-0.26 \pm 0.12$ & $\sim 172$ & 14 \\
\hline Kepler 296 & Kepler-296 e, $\mathrm{f}^{\mathrm{a}}$ & $4.2(+3.4,-1.6)$ & $-0.12 \pm 0.12$ & $\sim 226$ & 14 \\
\hline Kepler 436 & Kepler-436 $b^{\mathrm{a}}$ & $3.0(+7.7,-0.3)$ & $0.01 \pm 0.1$ & $\sim 618$ & 14 \\
\hline Kepler 437 & Kepler-437 $\mathrm{b}^{\mathrm{a}}$ & $2.9(+7.5,-0.3)$ & $0.00 \pm 0.1$ & $\sim 417$ & 14 \\
\hline Kepler 438 & Kepler-438 b & $4.4(+0.8,-0.7)$ & $0.16 \pm 0.14$ & $\sim 145$ & 14 \\
\hline Kepler 439 & Kepler-439 b ${ }^{a}$ & $7.2(+3.6,-3.9)$ & $0.02 \pm 0.1$ & $\sim 693$ & 14 \\
\hline Kepler 440 & Kepler-440 $b^{a}$ & $1.3(+0.6,-0.2)$ & $-0.3 \pm 0.15$ & $\sim 261$ & 14 \\
\hline Kepler 441 & Kepler-441 b & $1.9(+0.65,-0.4)$ & $-0.57 \pm 0.18$ & $\sim 284$ & 14 \\
\hline Kepler 442 & Kepler-442 b & $2.9(+8.1,-0.2)$ & $-0.37 \pm 0.1$ & $\sim 342$ & 14 \\
\hline Kepler 443 & Kepler- $443 b^{a}$ & $3.2(+7.5,-0.4)$ & $-0.01 \pm 0.1$ & $\sim 779$ & 14 \\
\hline KOI 4427 & KOI $4427 b^{a}$ & $3.6(+2.6,-1.3)$ & $-0.07 \pm 0.14$ & $\sim 240$ & 14 \\
\hline Kepler 174 & Kepler-174 d ${ }^{\mathrm{a}}$ & 7. \pm 4 & -0.556 & 360 & 15 \\
\hline Kepler 309 & Kepler-309 c $\mathrm{c}^{\mathrm{a}}$ & 1.5 & -0.415 & 581 & 16 \\
\hline Kepler 421 & Kepler-421 b & 4. \pm 0.8 & -0.25 & 320 & 15 \\
\hline Kepler 108 & Kepler-108 c & $8.9 \pm 3.7$ & -0.026 & 861 & 15 \\
\hline Kepler 397 & Kepler-397 c & $0.6 \pm 3.8$ & -0.035 & 1154 & 15 \\
\hline Kepler 90 & Kepler-90 h & $0.53 \pm 0.88$ & -0.17 & 835 & 15 \\
\hline Kepler 87 & Kepler-87 c & $0.5 \pm 3.7$ & -0.17 & 782 & 15 \\
\hline Kepler 69 & Kepler-69 c & $0.4 \pm 4.7$ & -0.29 & 360 & 15 \\
\hline Kepler 235 & Kepler-235 $\mathrm{e}^{\mathrm{a}}$ & 1.5 & 0.087 & 525 & 16 \\
\hline Kepler 283 & Kepler-283 c $\mathrm{c}^{\mathrm{a}}$ & 2.0 & -0.26 & 534.4 & 16 \\
\hline Kepler 298 & Kepler-298 d d $^{\mathrm{a}}$ & 1.5 & -0.121 & 474.3 & 16 \\
\hline EPIC 201367065 & EPIC $201367065 \mathrm{~d}$ & $2 \pm 1$ & $-0.32 \pm 0.13$ & 45 & 17 \\
\hline tau Ceti & tau Ceti e & 5.8 & $-0.55 \pm 0.05$ & 3.65 & 18 \\
\hline
\end{tabular}

${ }^{a}$ The radii of these planets are $>1.7$ Earth's, however, it is still too soon to exclude them from the list, according to Torres et al. (2015), since there are many uncertainties in the modelling of the transition from rocky to hydrogen/helium planets, and these planets may be rocky.

References to ages: 1. The Extrasolar Planet Encyclopaedia (http://exoplanet.eu); 2. Angalada-Escudé et al. (2012); 3. Anglada-Escudé et al. (2013); 4. Borucki et al. (2013); 5. Anglada-Escudé et al. (2014); 6. Mamajek \& Hillenbrand (2008); 7. Tuomi \& Anglada-Escudé (2013); 8. Open Exoplanet Catalogue (http://www.openexoplanetcatalogue.com); 9. Nordström et al. (2004); 10. Tuomi et al. (2013a); 11. Pepe et al. (2011); 12. Metcalfe (2013); 13. Wittenmyer et al. (2014); 14. Torres et al. (2015); 15. NASA Exoplanet Archive at http://exoplanetarchive.ipac.caltech.edu; 16. Gaidos (2013); 17. Crossfield et al. (2015); 18. Tuomi et al. (2013b).

The age of a planet is of primary importance for developing the future strategy of looking for life on PHPs. Since space programs are extremely expensive and require extensive valuable telescope time, it is crucial to know in advance which planets are more likely to host detectable life. Young planets will not have atmospheres abundant in products of photosynthetic processes, and many planets, though residing in the HZ, may not actually be habitable for life as we know it. For example, the host stars in the Degenerate Objects around Degenerate Objects (DODO) direct imaging search for sub-solar mass objects around white dwarfs (Hogan et al. 2009) are rather young with an average age of only $2.25 \mathrm{Gyr}$. The target star selection of the Darwin (ESA) mission is restricted to stars within $10-25 \mathrm{pc}$ (Kaltenegger \& Fridlund 2005), and two space missions that are currently under study, the NASA Transiting Exoplanet Survey Satellite (TESS) mission and ESA's PLAnetary Transits and Oscillations of stars (PLATO) mission, will only survey bright F, G, K stars and M stars within 50 pc (e.g., Lammer et al.
2013), sampling therefore only the thin Galactic disc stars young Pop I hosts. The main focus of Exoplanet Characterization Observatory (EChO) (Drossart et al. 2013) is the observation of hot Jupiter and hot Neptune planets, limited due to the mission lifetime constraints to bright nearby $\mathrm{M}$ stars (Tinetti et al. 2012). Most known habitable planets cannot have an existing complex biosphere although they may develop it in the future, because most currently known PHPs are found around relatively young Pop I stars. We feel that it is reasonable to fix a period of $\sim 4 \mathrm{Gyr}$ as the minimum necessary time for the formation of complex life forms under optimal conditions, as evidenced by the Earth's biosphere. Direct observations of planetary atmospheres in IR and sub-mm wavebands would be a promising method for tracing biogenesis. Planned future IR and sub-mm observatories could provide such observations (see the discussion in Section 'Observational prospects' below.).

In this context, we have undertaken the project of updating the catalogue of Nearby Habitable Systems (HabCat) 
constructed for SETI by Turnbull \& Tarter (2003a) for the search for potentially habitable hosts for complex life. A complete characterization of all the stars within a few hundred (or even a few tens of) parsecs, including their masses, ages, and whether they have planetary systems (including terrestrial planets), was not realizable at that time. Our aim was to find out the information on these stars: their ages and whether they have planets and if they could be potentially habitable.

To begin with, we have taken the HabCat II, a 'Near 100' subset - a list of the nearest 100 star systems of the original HabCat (Turnbull \& Tarter 2003b), as a basis for our project. These stars were scrutinized for information on their age, nearby planets etc., which were missing in the original catalogue but are important now due to their impact on selecting the targets for future space missions. Out of 100 nearby (within $10 \mathrm{pc}$ ) objects in the HabCat II, we have found the age data for 50 stars. This list is being cross-correlated with the Hypatia Catalogue, which is a project to find abundances for 50 elements, specifically bio-essential elements, for the stars in the HabCat (Hinkel et al. 2014). Our goal is to compile a list of the most probable planets that may allow future missions to search our neighbourhood for habitable/inhabited planets more efficiently. The preliminary result of this project is presented in Table A.2 in Appendix B.

\section{Old planetary systems}

\section{General census}

Most of the old planetary systems were discovered serendipitously. Only in 2009 were targeted surveys of metal-poor stars initiated (Setiawan et al. 2010). In spite of that, quite a few old ( $\gtrsim 9$ Gyr) planetary systems are currently known. Shchekinov et al. (2013) attempted to compile a list of such system (see their Table 1) on the basis of metallicity, considering stars $[\mathrm{Fe} / \mathrm{H}] \leq-0.6$. They, however, missed many previously known systems with ages determined by several different methods, including metallicity abundances, chromospheric activity, rotation and isochrones. Combining their table with other studies (Saffe et al. 2005; Haywood 2008 and latest updates of online exoplanet catalogues) brings the census of planetary systems with ages $\gtrsim 9$ Gyr to 116 planets (90 host stars; see Appendix A for the table of these systems). It is possible that the number of such hosts is much larger since we have counted only those stars where estimates from different methods were comparable. For example, in the list of NASA Exoplanet Archive candidates to PHPs, out of 62 hosts with estimated ages, 28 are older than $10 \mathrm{Gyr}$.

The majority of old planets were detected by the radial velocity method which is biased to detect preferentially massive planets due to a limited sensitivity. The continuously increasing precision of radial-velocity surveys may in future change this picture, and the first example of that is the detection in mid-2014 of the terrestrial planet ( $\sim 5$ Earth masses) orbiting extremely old (10-12 Gyr) Kapteyn's star (Anglada-Escudé et al. 2014). The most remarkable thing is that this planet lies in the HZ. The star also has another super-Earth outside the HZ.

\section{Potential habitability of old planetary systems}

The improved precision has also resulted in the rejection of three previously reported old planets HIP $13044 \mathrm{~b}$ and HIP 11952 b, c (e.g., Setiawan et al. 2010, 2012) as a genuine signal (Jones \& Jenkins 2014). However, it still leaves the number of old planets of at least 117 (92 hosts, see Table A.1 in the Appendix A) with 11 super-Earths (namely, Kepler-18 b; 55 Cnc e; Kapteyn's b, c; MOA-2007-BLG-192L b, OGLE-2005-BLG-390L b and five planets of Kepler-444) and all the rest gas giants, which do not fall into the category of habitable planets. However, because giant planets typically harbour multiple moons, the moons may be habitable and may even lie in the domain of a higher habitability, or even 'superhabitability' (Heller \& Armstrong 2014). For example, Schulze-Makuch et al. (2011) estimate the PHI for Jupiter to be only 0.4 , while it is around 0.65 for Titan. There are 33 potentially habitable exomoons with habitable surfaces listed by HEC (excluding possibility of subsurface life), which have on average ESI higher than the PHPs. Heller et al. (2014) have shown that the number of moons in the stellar HZ may even outnumber planets in these circumstellar zones, and that massive exomoons are potentially detectable with current technology (Heller 2014). Even though Population II (Pop II) stars are normally two order of magnitude less abundant in metals, they may harbour up to ten potentially habitable rocky Earth-size subsolar objects each (Shchekinov et al. 2013), either as planets or as moons orbiting gaseous giants. Planets can form at metallicities as low as $Z \sim 0.01 Z_{\odot}$ due to the centrifugal accumulation of dust (Shchekinov et al. 2013). However, Pop II stars could have formed in the metal-enriched pockets resulting from a non-perfect mixing in young galaxies when the Universe was as young as a few hundreds of Myr (Dedikov \& Shchekinov 2004; Vasiliev et al. 2009). They would be able to form planets in a traditional way, and our Galaxy may have a vast number of rocky planets residing in habitable zones. Such planets had longer time for developing biogenesis. Recently discovered five rocky planets orbiting 11.2 Gyr old star Kepler-444 (Campante et al. 2015) seems to confirm the previously suggested (Shchekinov et al. 2013) hypothesis.

Direct measurements of metallicities and abundance pattern in the early Universe have recently become possible with the discovery of extremely metal-poor (EMP) stars with metallicities as low as $10^{-5}$ of the solar value - these objects are believed to represent the population next after the Population III (Pop III) stars (Beers \& Christlieb 2005). The relative abundances observed in the EMP stars are shown to stem from the explosions of Pop III intermediate-mass SNe with an enhanced explosion energy about $5 \times 10^{51}$ erg (Umeda \& Nomoto 2005). These stars are also often found to be overabundant in CNO elements. Interestingly, their relative abundance (Aoki et al. 2006; Ito et al. 2013) is consistent with the abundance pattern of the Earth crust (Taylor \& McLennan 1995; Yanagi 2011) and the chemical composition of the human body (see, e.g., Nielsen 1997). 
Though Earth is rich in chemistry, living organisms use just a few of the available elements: C, N, O, H, P and S, in biological macromolecules: proteins, lipids and DNA, which can constitute up to $98 \%$ of an organisms' mass (e.g., Alberts et al. 2002). Apart from hydrogen, these 'biogenic' elements are all produced by the very first massive Pop III stars. Detection of substantial amount of $\mathrm{CO}$ and water in the spectrum of $z=6.149$ quasar SDSS J1148 + 5251 shows, for example, that at $\sim 800$ Myr after the Big Bang, all the ingredients for our carbonbased life were already present. The initial episode of metal enrichment is believed to have occurred when the Universe was about 500-700 $\mathrm{Ma}$ - the absorption spectra of high-redshift galaxies and quasars show significant amount of metals, in some cases up to 0.3 of the solar metallicity (e.g., Savaglio 2006; Finkelstein et al. 2013). The abundance pattern of heavy elements in this initial enrichment contains a copious amount of elements sufficient for rocky planets to form within the whole range of masses (Bromm et al. 1999; Abel et al. 2000; Clark et al. 2011; Stacy et al. 2011).

Therefore, planets formed in the early Universe and observed now as orbiting very old ( $\gtrsim 9$ Gyr) Pop II stars, may have developed and sustained life over the epochs when our Solar System had only started to form. In this way, the restricted use of six 'biogenic' elements may be considered as a fossil record of an ancient life - it is well known that at the molecular level, living organisms are strongly conservative. The general direction of the biological evolution is in the increase of complexity of species rather than (chemical) diversity (Mani 1991). For example, paradoxically, both oxygen and water are destructive to all forms of carbon-based life (e.g., Bengston 1994). The presence of water reduces the chance of constructing nucleic acids and most other macromolecules (Schulze-Makuch \& Irwin 2006). The toxic nature of oxygen necessitated the evolution of a complex respiratory metabolism, which again shows the strong chemical conservatism at the molecular level in that the living organisms developed the protection mechanisms to circumvent these problems rather than use other compounds.

\section{Observational prospects}

Recently, a 13.6 Gyr star was detected placing it as the oldest star in the Universe (SMSS J031300.36-670839.3, Keller et al. 2014); the age was estimated by its metallicity $[\mathrm{Fe} / \mathrm{H}] \leq-7.41$. In spite of that, this star, believed to have formed from the remnants of the first-generation $\mathrm{SN}$, was found to contain carbon, metals such as lithium, magnesium, calcium, and even methylidyne $(\mathrm{CH})$. It is quite possible that such stars have planets that are directly observable in micron wavelength range. Such EMP stars are known to have low masses and, as such, the orbiting planets could be seen directly in the IR.

The number of EMP stars is estimated to be about 250,000 within 500 pc in SDSS database (Aoki et al. 2006), so the mean distance between them is about 10 pc. If each EMP star hosts an Earth-size planet, the flux from the planet at a distance $d$ in the IR range $(\lambda \sim 10 \mu \mathrm{m})$ evaluated at the peak frequency
(Wien's law) $v_{\mathrm{T}}=\alpha k T / h$, is

$$
F_{v}^{\mathrm{pl}}=\sigma T_{\mathrm{pl}}^{4} \pi\left(\frac{R_{\mathrm{pl}}}{d}\right)^{2}
$$

we can rewrite this flux as

$$
F_{\nu}^{\mathrm{pl}}=0.73\left(\frac{T_{\mathrm{eq}}}{300 \mathrm{~K}}\right)^{3}\left(\frac{d}{10 \mathrm{pc}}\right)^{-2}\left(\frac{R}{R_{\mathrm{E}}}\right)^{2} \mathrm{mJy},
$$

where $T_{\text {eq }}$ is the equilibrium temperature of a planet and $R$ is its radius. For the Sun/Earth system, the ratio of the fluxes at a distance of $10 \mathrm{pc}$ is

$$
\frac{F_{v}^{\mathrm{pl}}}{F_{*}}=\frac{T_{\mathrm{E}}}{T_{\odot}}\left(\frac{R_{\mathrm{E}}}{R_{\odot}}\right)^{2} \sim 4 \times 10^{-6} .
$$

However, if we consider a super-Earth with $M \sim 5 M_{\mathrm{E}}$, $R \sim 2 R_{\mathrm{E}}$ and $T_{\mathrm{eq}}=300 \mathrm{~K}$, orbiting the star with $T=3000 \mathrm{~K}$ and $R \sim 0.1 R_{\odot}-$ an $M$ dwarf, we get an improvement of

$$
\frac{F_{v}^{\mathrm{pl}}}{F_{*}}=3.4 \times 10^{-3}
$$

It seems challenging to detect such a weak contribution to a total flux from a planet even in the IR. There is, however, a possibility to distinguish the emission from the planet in IR molecular features, such as $\mathrm{CH}_{4}$ or $\mathrm{O}_{2}$, tracing either initiated biogenesis or developed metabolism. Detection of direct IR emission from $\mathrm{O}_{2}$ on exoplanets going through the initial epoch of biogenesis, or which are already at a stage with developed biota, was discussed in Churchill \& Kasting (2000) and Rodler \& Lopez-Morales (2014), respectively. Rich IR to sub-mm spectra of methane (Niederer 2012; Hilico et al. 1987) also allow to optimistically view the future detection of this biosignature. Even at the low temperatures of EMP stars, $T_{*} \sim 3000 \mathrm{~K}$, these molecules are unlikely to survive in sufficient amount in their atmospheres. Therefore, if such emission is observed from an EMP star, it should be considered as a direct indication of an orbiting rocky planet that has already entered the habitable epoch with growing PHI (equation (6)). The most promising way to identify habitable (inhabited) planets seems to look for simultaneous presence of water, $\mathrm{O}_{2}, \mathrm{O}_{3}$, $\mathrm{CH}_{4}$ and $\mathrm{N}_{2} \mathrm{O}$ in atmospheric spectra (e.g., Selsis et al. 2002; Kaltenegger et al. 2007; Kiang et al. 2007). Though such observations can be used to detect planets with highly developed habitability orbiting old EMP stars, the expected fluxes in the IR are still below current sensitivity limits and might be only possible in the future. For example, the future Millimetron space observatory planned for launching in next decade (estimated launch 2025) will have the detection limit of $0.1 \mu \mathrm{Jy}$ in $1 \mathrm{~h}$ observation in 50-300 $\mu \mathrm{m}$ range (Kardashev et al. 2014). A molecular $\mathrm{CH}_{4}$ absorptions at $\sim 50 \mu \mathrm{m}$ can be detected by Millimetron in $3 \mathrm{~h}$ observations (Equation (10)) if a nearby (within $10 \mathrm{pc}$ ) habitable super-Earth planet transits an M-dwarf.

\section{Summary}

- Age of a planet is an essential attribute of habitability along with such other factors as liquid water (or an 
equivalent solvent), rocky mantle, appropriate temperature, extended atmosphere, and so forth. The knowledge of the age of a 'habitable' planet is an important factor in developing a strategy to search for complex (developed) life.

- Nearly half of the confirmed PHPs are young (with ages less than $~ 3.5 \mathrm{Gyr}$ ) and may not have had enough time for evolution of sufficiently complex life capable of changing its environment on a planetary scale;

- Planets do exist around old Pop II stars, and recently discovered EMP stars (belonging presumably to an intermediate Pop II.5) are good candidates for direct detection of orbiting planets in the IR and sub-mm wavelengths. Though currently only very few such PHPs are known, old giant planets may have habitable worlds in the form of orbiting moons.

- IR and sub-mm observations of terrestrial planets orbiting low-mass old stars are a promising way to trace biogenetic evolution on exoplanets in the solar neighbourhood.

\section{Acknowledgements}

Y. S. acknowledges the hospitality of RRI and IIA, Bangalore, when this work has been initiated. The authors thank Tarun Deep Saini for his useful comments, IIA $\mathrm{Ph}$. D. student A. G. Sreejith for help with graphics and IIA internship student Anuj Jaiswal for his contribution in the project of updating the HabCat. The authors also thank the referees for their valuable comments which led to considerable improvement in the paper. This research has made use of the Extrasolar Planets Encyclopaedia at http://www.exoplanet.eu, Exoplanets Data Explorer at http://exoplanets.org, NASA Exoplanet Archive at http:// exoplanetarchive.ipac.caltech.edu and NASA Astrophysics Data System Abstract Service.

\section{References}

Abel, T., Bryan, G.L. \& Norman, M.L. (2000). The formation and fragmentation of primordial molecular clouds. Astrophys. J. 540, 39.

Alberts, B. et al. (2002). Molecular Biology of the Cell, 4th edn. Garland Science, New York.

Anbar, A.D., Duan, Y., Lyons, T.W., Arnold, G.L., Kendall, B., Creaser, R.A., Kaufman, A.J., Gordon, G.W., Scott, C., Garvin, J. \& Buick, R. (2007). A whiff of oxygen before the great oxidation event? Science 317, 1903.

Anglada-Escudé, G. et al. (2012). A planetary system around the nearby M Dwarf GJ $667 \mathrm{C}$ with at least one super-earth in its habitable zone. Astrophys. J. Lett. 751, L16.

Anglada-Escudé, G. et al. (2013). A dynamically-packed planetary system around GJ $667 \mathrm{C}$ with three super-Earths in its habitable zone. Astron. Astrophys. 556, A126.

Anglada-Escudé, G. et al. (2014). Two planets around Kapteyn's star: a cold and a temperate super-Earth orbiting the nearest halo red dwarf. Mon. Not. R. Astron. Soc. 443, L89.

Aoki, W. et al. (2006). HE 1327-2326, an unevolved star with $[\mathrm{Fe} / \mathrm{H}]$ $<-5.0$. I. A comprehensive abundance analysis. Astrophys. J. 639, 897.
Beers, T.C. \& Christlieb, N. (2005). The discovery and analysis of very metal-poor stars in the galaxy. Ann. Rev. Astron. Astrophys. 43, 531.

Bengston, S. (Ed.) (1994). Early Life on Earth. Nobel Symp. Proc. Series. Columbia University Press, New York. ISBN-10\#0231080883.

Benson, A.A., Bassham, J.A., Calvin, M., Goudate, T.C., Haas, U.A. \& Stepka, W. (1950). The path of carbon in photosynthesis. 5. Paper chromatography and radioautography of the products. J. Am. Chem. Soc. 12, 1710.

Brack, A. et al. (2010). Origin and evolution of life on terrestrial planets. Astrobiology 10, 69-76.

Borucki, W.J. et al. (2013). Kepler-62: a five-planet system with planets of 1.4 and 1.6 Earth radii in the habitable zone. Science 340, 587-590.

Bouwens, R.J., Illingworth, G.D., Franx, M. \& Ford, H. (2007). UV luminosity functions at z 4, 5, and 6 from the hubble ultra deep field and other deep hubble space telescope ACS fields: evolution and star formation history. Astrophys. J. 670, 928.

Bromm, V., Coppi, P.S. \& Larson, R.B. (1999). Forming the first stars in the universe: the fragmentation of primordial gas. Astrophys. J. 527, L5.

Buchhave, L.A., Bizzarro, M., Latham, D.W., Sasselov, D., Cochran, W.D., Endl, M., Isaacson, H., Juncher, D. \& Marcy, G.W. (2014). Three regimes of extrasolar planet radius inferred from host star metallicities. Nature $\mathbf{5 0 9}$, 593.

Campante, T.L. et al. (2015). An ancient extrasolar system with five sub-earth-size planets. Astrophys. J. 799, 170.

Carraro, G., Geisler, D., Villanova, S., Frinchaboy, P.M. \& Majewski, S.R. (2007). Old open clusters in the outer Galactic disk. Astron. Astrophys. 476, 217-227.

Carter, B. (1983). The anthropic principle and its implications for biological evolution. Phil. Trans. R. Soc. Lond. A 310, 347-363.

Catling, D.C., Glein, C.R., Zahnle, K.J. \& McKay, C.P. (2005). Why $\mathrm{O}_{2}$ is required by complex life on habitable planets and the concept of planetary "oxygenation time". Astrobiology 5, 415-438.

Chen, L., Hou, J.L. \& Wang, J.J. (2003). On the galactic disk metallicity distribution from open clusters. I. New catalogs and abundance gradient. Astronom. J. 125, 1397.

Churchill, D. \& Kasting, J.F. (2000). Nitrous oxide in the early atmosphere: a marker for life? In Proc. Conf. 'Darwin and Astronomy - the Infrared Space Interferometer', Stockholm, Sweden, 17-19 November 1999. European Space Agency, Noordwijk, The Netherlands, ESA SP 451, p. 183.

Chyba, C.F. \& Hand, K.P. (2005). Astrobiology: the study of the living universe. Annu. Rev. Astron. Astrophys. 43, 31 .

Clark, P.C., Glover, S.C.O., Smith, R.J., Greif, T.H., Klessen, R.S. \& Bromm, V. (2011). The formation and fragmentation of disks around primordial protostars. Science 331, 1040.

Crick, F.H.C. \& Orgel, L.E. (1973). Prebiotic activation processes. Icarus 19, 341.

Crossfield, I.J.M. et al. (2015). A nearby M star with three transiting super-Earths discovered by K2. Astrophys. J. 804, 10.

da Silva, L., Girardi, L., Pasquini, L., Setiawan, J., von der Lühe, O., de Medeiros, J.R., Hatzes, A., Döllinger, M.P. \& Weiss, A. (2006). Basic physical parameters of a selected sample of evolved stars. Astron. Astrophys. 458, 609-623.

Davies, P.C.W., Benner, S.A., Cleland, C.E., Lineweaver, C.H., McKay, C.P. \& Wolfe-Simon, F. (2009). Signatures of a shadow biosphere. Astrobiology 9, 241.

Dedikov, S.Yu. \& Shchekinov, Yu.A. (2004). Mixing of metals during stripping of galactic gaseous halos. Astrol. Rep. 48, 9.

Döllinger, M.P., Hatzes, A.P., Pasquini, L., Guenther, E.W., Hartmann, M. \& Girardi, L. (2000). Planetary companion candidates around the K giant stars 42 Draconis and HD 139 357. Astron. Astrophys. 499, 935-942.

Domagal-Goldman, S.D., Meadows, V.S., Claire, M.W. \& Kasting, J.F. (2011). Using biogenic sulfur gases as remotely detectable biosignatures on anoxic planets. Astrobiology 11, 419-441.

Drossart, P. et al. (2013). The Exoplanet Characterisation Observatory (EChO): an ESA mission to characterize exoplanets. AAS/Division for Planetary Sciences Meeting Abstracts, 45, \#211.25.

Etiope, G. \& Sherwood Lollar, B. (2013). Abiotic methane on earth. Rev. Geophys. 51, 276-299. 
Farquhar, G.D., von Caemmerer, S. \& Berry, J.A. (1980). A biochemical model of photosynthetic $\mathrm{CO}_{2}$ assimilation in leaves of $\mathrm{C} 3$ species. Planta 149, 78.

Finkelstein, S.L. et al. (2013). A galaxy rapidly forming stars 700 million years after the Big Bang at redshift 7.51. Nature 502, 524.

Fomina, I., Biel, K. (2014). Photosynthetic carbon metabolism: strategy of adaptation. In Contemporary Problems of Photosynthesis, vol. 2, ed. Allakhverdiev, S.I., Rubin, A.B. and Shuvalov, V.A., pp. 415-483. Institute of Computer Science, Izhevsk, Moscow [in Russian].

France, K. et al. (2013). The ultraviolet radiation environment around M dwarf exoplanet host stars. Astrophys. J. 763, 149.

Gaidos, E. (2013). Candidate planets in the habitable zones of Kepler stars. Astrophys. J. 770, 90

Haywood, M. (2008). A peculiarity of metal-poor stars with planets? Astron. Astrophys. 482, 673-676.

Heger, A. \& Woosley, S.E. (2002). The nucleosynthetic signature of population III. Astrophys. J. 567, 532.

Heller, R. (2014). Detecting extrasolar moons akin to solar system satellites with an orbital sampling effect. Astrophys. J. 787, 14.

Heller, R. \& Armstrong, J. (2014). Superhabitable worlds. Astrobiology 14, 50.

Heller, R. et al. (2014). Formation, habitability, and detection of extrasolar moons. Astrobiology 14, 798-835.

Hikosaka, K., Ishikawa, K., Borjigidai, A., Muller, O. \& Onoda, Y. (2006). Temperature acclimation of photosynthesis: mechanisms involved in the changes in temperature dependence of photosynthetic rate. J. Exp. Bot. 57, 291.

Hilico, J.H., Loete, M. \& Champion, J.P. (1987). The millimiter-wave spectrum of Methane. J. Mol. Spectrosc. 122, 381

Hinkel, N.R., Timmes, F.X., Young, P.A., Pagano, M.D. \& Turnbull, M.C. (2014). Stellar abundances in the solar neighborhood: the hypatia catalog. Astronom. J. 148, 54.

Hogan, E., Burleigh, M.R. \& Clarke, F.J. (2009). The DODO survey - II. A Gemini direct imaging search for substellar and planetary mass companions around nearby equatorial and Northern hemisphere white dwarfs. Mon. Not. R. Astron. Soc. 396, 2074.

Huang, S.-S. (1959). The problem of life in the universe and the mode of star formation. Publ. Astron. Soc. Pacific 71, 421-424.

Irwin, L.N., Méndez, A., Fairén, A.G. \& Schulze-Makuch, D. (2014). Assessing the possibility of biological complexity on other worlds, with an estimate of the occurrence of complex life in the milky way galaxy. Challenges 5, 159 .

Ito, H., Aoki, W., Beers, T.C., Tominaga, N., Honda, S. \& Carollo, D. (2013). Chemical analysis of the ninth magnitude carbon-enhanced metal-poor star BD+44-493. Astrophys. J. 773, 33.

Jones, M.I. \& Jenkins, J.S. (2014). No evidence of the planet orbiting the extremely metal-poor extragalactic star HIP 13044. Astron. Astrophys. 562, A129.

Kaltenegger, L. \& Fridlund, M. (2005). The Darwin mission: search for extrasolar planets. Adv. Space Res. 36, 1114-1122.

Kaltenegger, L., Traub, W.A. \& Jucks, K.W. (2007). Spectral evolution of an earth-like planet. Astrophys. J. 658, 598.

Kardashev, N.S. et al. (2014). Review of scientific topics for the millimetron space observatory. Phys. - Usp. 57, 1199-1228.

Kasting, J. (1993). Earth's early atmosphere. Science 259, 920.

Kasting, J.F., Kopparapu, R., Ramirez, R.M. \& Harman, C.E. (2014). Remote life-detection criteria, habitable zone boundaries, and the frequency of Earth-like planets around M and late K stars. Proc. Natl. Acad. Sci. USA 111, 12641-12646.

Kharecha, P., Kasting, J. \& Siefert, J. (2005). A coupled atmosphere-ecosystem model of the early Archean Earth. Geobiology 3, 53-76.

Kiang, N.Y., Segura, A., Tinetti, G., Govindjee, Blankenship, R.E., Cohen, M., Siefert, J., Crisp, D. \& Meadows, V.S. (2007). Spectral signatures of photosynthesis. II. Coevolution with other stars and the atmosphere on extrasolar worlds. Astrobiology 7, 252.

Keller, S.C. et al. (2014). A single low-energy, iron-poor supernova as the source of metals in the star SMSS J031300.36-670839.3. Nature 506, 463.

Kenrick, P. \& Crane, P.R. (1997). The origin and early evolution of plants on land. Nature 389, 33-39.
Kepner, T. (2007). ... And Remote from Neighbours. Wilder Publications, LLC, Radford, VA. ISBN 10:1-934451-02-9.

Lammer, H. et al. (2009). What makes a planet habitable? Astron Astrophys. Rev. 17, 181.

Lammer, H. et al. (2013). The science of exoplanets and their systems. Astrobiology 13, 793-813.

Lin, H.W., Gonzalez Abad, G. \& Loeb, A. (2014). Detecting industrial pollution in the atmospheres of earth-like exoplanets. Astrophys. J. Lett. 792, LL7.

Lisse, C.M., Wyatt, M.C., Chen, C.H., Morlok, A., Watson, D.M., Manoj, P., Sheehan, P., Currie, T.M., Thebault, P. \& Sitko, M.L. (2012). Spitzer evidence for a late-heavy bombardment and the formation of ureilites in eta corvi at $\sim 1$ Gyr. Astrophys. J. 747, 93L.

Lyons, T.W. \& Reinhard, C.T. (2011). Earth science: sea change for the rise of oxygen, Nature 478, 194-195.

Maher, K.A. \& Stevenson, D.J. (1988). Impact frustration of the origin of life. Nature 331, 612.

Mamajek, E.E. \& Hillenbrand, L.A. (2008). Improved age estimation for solar-type dwarfs using activity-rotation diagnostics. Astrophys. J. 687, 1264-1293.

Mani, G.S. (1991). In Evolutionary Theories of Economic and Technological Change - Present Status and Future Prospects, ed. Saviotti, P.P. and Metcalfe, J.S., pp. 31-57. Harwood Academic Publishers, UK.

Melosh, H.J. \& Vickery, A.M. (1989). Impact erosion of the primordial atmosphere of Mars. Nature 338, 487.

Méndez, A., González, Z., Jimenez, S.A., Pérez, W. \& Bracero, K. (2013). The Visible Paleo-Earth Project: A look from space to the evolution of a habitable planet. [in preparation]. http://phl.upr.edu/projects/visual-paleoearth

Metcalfe, T.S. (2013). Asteroseismology - A Tool for Characterizing Exoplanet Host Star. Invited talk at NASA Exoplanet Exploration Program Analysis Group, Long Beach, CA, USA.

Moynier, F., Koeberl, C., Quitté, G. \& Telouk, P. (2009). A tungsten isotope approach to search for meteoritic components in terrestrial impact rocks. Earth Planet Sci. Lett. 286, 35.

Niederer, J.M.G. (2012). The Infrared Spectrum of Methane. Dissertation ETH Nr. 19829, Eidgenössische Technische Hochschule Zürich (ETH Zürich), Zürich, Verlag Dr. Hut, München, DOI:10.3929/ ethz-a-007316862.

Nielsen, F.H. (1997). Beyond copper, iodine, selenium and zinc: other elements that will be found important in human nutrition by the year 2000. In Proc. of 9th Int. Symp. on Trace Elements in Man and Animals (TEMA-9), Banff, Alberta, Canada, 19-24 May 1996, ed. Fischer, P.W. F., L'Abbé, M.R., Cockell, K.A. and Gibson, R.S., pp. 653-656. NRC Research Press, Ottawa.

Nordström, B., Mayor, M., Andersen, J., Holmberg, J., Pont, F., Jørgensen, B.R., Olsen, E.H., Udry, S. \& Mowlavi, N. (2004). The GenevaCopenhagen survey of the Solar neighbourhood. Ages, metallicities, and kinematic properties of $14000 \mathrm{~F}$ and $\mathrm{G}$ dwarfs. Astron. Astrophys. 418, 989-1019.

Pepe, F., Lovis, C., Ségransan, D., Benz, W., Bouchy, F., Dumusque, X., Mayor, M., Queloz, D., Santos, N.C. \& Udry, S. (2011). The HARPS search for Earth-like planets in the habitable zone. Astron. Astrophys. 534, A58.

Petigura, E.A., Howard, A.W. \& Marcy, G.W. (2013). Prevalence of Earth-size planets orbiting Sun-like stars. Proc. Natl. Acad. Sci. USA 110, 19273.

Robbins, S.J. \& Hynek, B.M. (2012). Impact History of Large Bollides at Mars: Implications for the Late Heavy Bombardment and Isochron Uncertainties. 43rd Lunar and Planetary Science Conference, \#1649.

Rodler, F. \& Lopez-Morales, M. (2014). Feasibility studies for the detection of $\mathrm{O}_{2}$ in an earth-like exoplanet. Astrophys. J. 781, 54.

Saffe, C., Gómez, M. \& Chavero, C. (2005). On the ages of exoplanet host stars. Astron. Astrophys. 443, 609-626.

Sagan, C. (1974). The origin of life in a cosmic context. Orig. Life Evol. Biosph. 5, 497.

Sagan, C., Thompson, W.R., Carlson, R., Gurnett, D. \& Hord, C. (1993). A search for life on Earth from the Galileo spacecraft. Nature 365, 715.

Santos, N.C. et al. (2010). The HARPS search for southern extra-solar planets. XXI. Three new giant planets orbiting the metal-poor stars HD 5388, HD 181720, and HD 190984. Astron. Astrophys. 512, AA47. 
Savaglio, S. (2006). GRBs as cosmological probes - cosmic chemical evolution. New J. Phys. 8, 195.

Schaefer, L. \& Sasselov, D. (2015). The persistence of oceans on earth-like planets: insights from the deep-water cycle. Astrophysical J. 801:40

Schoenberg, R., Kamber, B.S., Collerson, K.D. \& Moorbath, S. (2002). Tungsten isotope evidence from $\sim 3.8-\mathrm{Gyr}$ metamorphosed sediments for early meteorite bombardment of the Earth. Nature 418, 403.

Schulze-Makuch, D. \& Irwin, L.N. (2006). The prospect of alien life in exotic forms on other worlds. Naturwissenschaften 93, 155-172.

Schulze-Makuch, D., Méndez, A., Fairén, A.D., von Paris, P., Turse, C., Boyer, G., Davila, A.F., António, M.R.D.S., Catling, D. \& Irwin, L.N. (2011). A two-tiered approach to assessing the habitability of exoplanets. Astrobiology 11, 1041-1052.

Seager, S., Schrenk, M. \& Bains, W. (2012). An astrophysical view of Earth-based metabollic biosignature gases. Astrobiology 12, 61-82.

Selsis, F., Despois, D. \& Parisot, J.P. (2002). Signature of life on exoplanets: can Darwin produce false positive detections? Astron. Astrophys. 388, 985.

Setiawan, J., Klement, R.J., Henning, Th., Rix, H.-W., Rochau, B., Rodmann, J. \& Schulze-Hartung, T. (2010). Giant planet around a metal-poor star of extragalactic origin. Science 330, 1642.

Setiawan, J., Roccatagliata, V., Fedele, D., Henning, Th., Pasquali, A., Rodríguez-Ledesma, M.V., Caffau, E.; Seemann, U. \& Klement, R.J. (2012). Planetary companions around the metal-poor star HIP 11952. Astron. Astrophys. 540, 141.

Schindler, T.L. \& Kasting, J.F. (2000). Synthetic spectra of simulated terrestrial atmospheres containing possible biomarker gases. Icarus $\mathbf{1 4 5}$, 262-271.

Shchekinov, Yu., Safonova, M. \& Murthy, J. (2013). Planets in the early universe. Astrophys. Space Sci. 346, 31-40.

Shizgal, B.D. \& Arkos, G.G. (1996). Nonthermal escape of the atmospheres of Venus, Earth, and Mars. Rev. Geophys. 34, 483.

Solomatov, V.S. (2000). Fluid dynamics of a terrestrial Magma Ocean. In Origin of the Earth and Moon, ed. Canup, R.M. et al., pp. 323-338. University Arizona Press, Tucson.

Song, I., Zuckerman, B., Weinberger, A.J. \& Becklin, E.E. (2005). Extreme collisions between planetesimals as the origin of warm dust around a Sun-like star. Nature 436, 363.

Stacy, A., Bromm, V. \& Loeb, A. (2011). Rotation speed of the first stars. Mon. Not. R. Astron. Soc. 413, 543.

Taylor, S.R. \& McLennan, S.M. (1995). Rev. Geophys. 33, 241.

Tinetti, G. et al. (2012). EChO. Exoplanet characterisation observatory. Exp. Astron. 34, 311-353.
Toole, G. \& Toole, S. (1997). Advance Human and Social Biology. Stanley Thornes Ltd., Cheltenham.

Torres, G. et al. (2015). Validation of twelve small Kepler transiting planets in the habitable zone. Astrophys. J. 800, 99.

Tuomi, M. \& Anglada-Escudé, G. (2013). Up to four planets around the M dwarf GJ 163. Sensitivity of Bayesian planet detection criteria to prior choice. Astron. Astrophys. 556, A111.

Tuomi, M., Anglada-Escudé, G., Gerlach, E., Jones, H.R.A., Reiners, A., Rivera, E.J., Vogt, S.S. \& Butler, R.P. (2013a). Habitable-zone super-Earth candidate in a six-planet system around the K2.5V star HD 40307. Astron. Astrophys. 549, A48.

Tuomi, M. et al. (2013b). Signals embedded in the radial velocity noise. Periodic variations in the tau Ceti velocities. Astron. Astrophys. 551, A79.

Turnbull, M.C. \& Tarter, J.C. (2003a). Target selection for SETI. I. A catalog of nearby habitable stellar systems. Astrophys. J. Suppl. 145, 181.

Turnbull, M. \& Tarter, J. (2003b). Target selection for SETI. II. Tycho-2 Dwarfs, old open clusters, and the nearest 100 stars. Astrophys. J. Suppl. 149, 423.

Tutukov, A.V. \& Fedorova, A.V. (2012). Formation of planets during the evolution of single and binary stars. Astrol. Rep. 56, 305.

Umeda, H. \& Nomoto, K. (2005). Variations in the abundance pattern of extremely metal-poor stars and nucleosynthesis in population III supernovae. Astrophys. J. 619, 427.

Vasiliev, E.O., Dedikov, S. \& Shchekinov, Yu. A. (2009). Chemical inhomogeneity of the post-reionization universe. Astrophys. Bull. 64, 317.

von Braun, K. et al. (2011). 55 Cancri: stellar astrophysical parameters, a planet in the habitable zone, and implications for the radius of a transiting super-earth. Astrophys. J. 740, 49.

Webster, C.R. et al. (2013). Isotope ratios of H, C, and $\mathrm{O}$ in $\mathrm{CO} 2$ and $\mathrm{H} 2 \mathrm{O}$ of the Martian atmosphere. Science 341, 260.

Wille, M., Kramers, J.D., Nägler, T.F., Beukes, N.J., Schröder, S., Meisel, Th., Lacassie, J.P. \& Voegelin, A.R. (2007). Evidence for a gradual rise of oxygen between 2.6 and $2.5 \mathrm{Ga}$ from Mo isotopes and Re-PGE signatures in shales. Geochim. Cosmochim. Acta 71, 2417-2435.

Wittenmyer, R.A. et al. (2014). GJ 832c: a super-earth in the habitable zone. Astrophys. J. 791, 114.

Wyatt, M.C., Smith, R., Greaves, J.S., Beichman, C.A., Bryden, G. \& Lisse, C.M. (2007). Transience of hot dust around sun-like stars. Astrophys. $J$. 658, 569.

Yanagi, T. (2011). Arc Volcano in Japan, Lecture Notes in Earth Sciences. Springer-Verlag, Berlin, Heidelberg.

\section{Appendix A. The list of stars with estimated ages $\geq 9$ Gyr.}

Table A.1. Stars with measuredlestimated masses of $\geq 9$ Gyr

\begin{tabular}{|c|c|c|c|}
\hline Star name & Age (Gyr) & Planets & Refs, notes \\
\hline $16 \mathrm{CygB}=\mathrm{HD} 217014$ & $9-10$ & $2.3 \mathrm{~J}$ & Saffe et al. (2005), isoch, Li \\
\hline GJ86 = HR637 & 12.5 & $4 \mathrm{~J}$ & Saffe et al. (2005), Fe/H \\
\hline rho $\mathrm{Crb}=\mathrm{HD} 143761$ & $11.9-12.1$ & $1 \mathrm{~J}$ & Saffe et al. (2005), isoch, $\mathrm{Fe} / \mathrm{H}$ \\
\hline HD4208 & 12.4 & $0.8 \mathrm{~J}$ & Saffe et al. (2005), Fe/H \\
\hline HD16141 = 79Ceti & 11.2 & $\mathrm{~S}=0.2 \mathrm{~J}$ & Saffe et al. (2005), isoch \\
\hline HD41004A & 9.5 & $>2.5 \mathrm{~J}$ & Saffe et al. (2005), Fe/H \\
\hline HD45350 & 12.6 & $\geq 2 \mathrm{~J}$ & Saffe et al. (2005), isoch \\
\hline HD65216 & 10.2 & $>1 \mathrm{~J}$ & Saffe et al. (2005), Fe/H \\
\hline HD73526 & 10.3 & $\mathrm{~b}>2 \mathrm{~J} ; \mathrm{c}>2.3 \mathrm{~J}$ & Saffe et al. (2005), isoch \\
\hline HD76700 & 11.5 & hot $>0.1 \mathrm{~J}$ & Saffe et al. (2005), isoch \\
\hline HD89307 & 12.2 & $\mathrm{~J}$ & Saffe et al. (2005), Fe/H \\
\hline HD108874 & $10.7-14.1$ & $\mathrm{~b}, \mathrm{c}>1 \mathrm{~J}$ & Saffe et al. (2005), isoch \\
\hline HD114386 & 9.2 & $1.2 \mathrm{~J}$ & Saffe et al. (2005), Fe/H \\
\hline HD114729 & $11.9-12.5$ & $1 \mathrm{~J}$ & Saffe et al. (2005), isoch \\
\hline HD134987 & 11.1 & $\mathrm{~b}=0.8 \mathrm{~J} ; \mathrm{c}=1.5 \mathrm{~J}$ & Saffe et al. (2005), isoch \\
\hline HD142022 & $9.4-17.2$ & $>4.47 \mathrm{~J}$ & Saffe et al. (2005), isoch \\
\hline HD154857 & 13.1 & 2 giants & Saffe et al. (2005), Fe/H \\
\hline HD162020 & 9.5 & $14.4 \mathrm{~J}$ & Saffe et al. (2005), Fe/H \\
\hline
\end{tabular}




\begin{tabular}{|c|c|c|c|}
\hline Star name & Age (Gyr) & Planets & Refs, notes \\
\hline HD168443 & 10.6 & $\mathrm{~b}>7.5 \mathrm{~J} ; \mathrm{c}>17.5 \mathrm{~J}$ & Saffe et al. (2005), isoch \\
\hline HD168746 & $9.2-16$ & gas giant & Saffe et al. (2005), isoch, $\mathrm{Fe} / \mathrm{H}$ \\
\hline HD190228 & 12.5 & giant & Saffe et al. (2005), Fe/H \\
\hline HD195019 & 10.6 & $3.7 \mathrm{~J}$ & Saffe et al. (2005), isoch \\
\hline HD208487 & 10.8 & $1 \mathrm{~J}$ & Saffe et al. (2005), Fe/H \\
\hline HD216437 & 8.7 & $2 \mathrm{~J}$ & Saffe et al. (2005), isoch \\
\hline HD217107 & $9.5-9.9$ & $1 \mathrm{~J}$ & Kepner (2007) \\
\hline HD181720 = HIP95262 & $9.4-12.1$ & Gas giant & Santos et al. (2010) HAPRS \\
\hline HD4308 = HIP3497 & 11.5 & $\mathrm{~N}=14 \mathrm{E}$ & Haywood (2008) \\
\hline HD6434 = HIP5054 & 10.4 & $>0.4 \mathrm{~J}$ & Haywood (2008) \\
\hline HD37124 = HIP26381 & 14.7 & $\mathrm{~b}, \mathrm{c}, \mathrm{d} \geq 0.7 \mathrm{~J}$ & Haywood (2008) \\
\hline HD47536 = HIP31688 & $9.33 \pm 1.88$ & $\mathrm{~b}=5 \mathrm{~J}, \mathrm{c}=7 \mathrm{~J}$ & Haywood (2008), da Silva et al. (2006) \\
\hline HD111232 = HIP62534 & 12.0 & $>7 \mathrm{~J}$ & Haywood (2008) \\
\hline HD114762 = HIP64426 & 12.4 & $>11 \mathrm{~J}$ & Haywood (2008) \\
\hline Kapteyn's & $10-12$ & $\mathrm{~b}, \mathrm{c}=\mathrm{sE}+\mathrm{sE}$ & Haywood (2008) \\
\hline PSR-B1620-26 (M4) & $12.8 \pm 2.6$ & $2.5 \mathrm{~J}$ & SSM \\
\hline BD20-2457 & 12.7 & $\mathrm{~b}=12.47 \mathrm{~J}, \mathrm{c}=21.42 \mathrm{~J}$ & SSM \\
\hline HD155358 & 11.9 & $\mathrm{~b}=0.85 \mathrm{~J}, \mathrm{c}=0.82 \mathrm{~J}$ & SSM \\
\hline HAT-P-26 & 9 & $8 \mathbf{J}$ & exoplanets.eu \\
\hline HD102365 & 9 & $N=16 E$ & Haywood (2008) \\
\hline HD96063 & 9 & $0.9 \mathrm{~J}$ & Haywood (2008) \\
\hline HD103197 & 9.1 & Gas giant, $31 \mathrm{E}$ & Haywood (2008) \\
\hline HD154672 & 9.28 & $5 \mathrm{~J}$ & Haywood (2008) \\
\hline KOI-1257a & 9.3 & $1.45 \mathrm{~J}$ & Haywood (2008) \\
\hline HD47536 & 9.33 & $\mathrm{~b}=5 \mathrm{~J}, \mathrm{c}=7 \mathrm{~J}$ & Haywood (2008) \\
\hline HD4203 & 9.41 & $\mathrm{~b}=1.2 \mathrm{~J}, \mathrm{c}=2 \mathrm{~J}$ & Haywood (2008) \\
\hline 42Dra $=$ hd 170693 & 9.49 & $\sim 4 \mathrm{~J}$ & Döllinger et al. (2000) \\
\hline HD11964 & 9.56 & $\mathrm{~b}=0.1 \mathrm{~J}(\mathrm{~N}), \mathrm{c}=0.6 \mathrm{~J}$ & Haywood (2008) \\
\hline HD88133 & 9.56 & $>0.3 \mathrm{~J}$ & Haywood (2008) \\
\hline HATS-2 & 9.7 & $1.3 \mathrm{~J}$ & Haywood (2008) \\
\hline HD87883 & 9.8 & $>1.8 \mathrm{~J}$ & Haywood (2008) \\
\hline Kepler-46 & 9.9 & $\mathrm{~b}=\mathrm{S}, \mathrm{c}=0.37 \mathrm{~J}$ & Haywood (2008) \\
\hline Kepler-18 & 10 & $6.9 \mathrm{E}=\mathrm{sE} ; 17 \mathrm{E}=\mathrm{N} ; 16 \mathrm{E}=\mathrm{N}$ & Haywood (2008) \\
\hline V391Peg & 10 & $>3.2 \mathrm{~J}$ & Haywood (2008) \\
\hline HAT-P-38 & 10.1 & $0.27 \mathrm{~J}=\mathrm{S}$ & Haywood (2008) \\
\hline $55 \mathrm{Cnc}=\mathrm{HD} 75732$ & 10.2 & $0.8 \mathrm{~J}=\mathrm{S},>0.17 \mathrm{~J}=\mathrm{S}, 3.8 \mathrm{~J}, 8.63 \mathrm{E}=\mathrm{sE},>0.155 \mathrm{~J}=\mathrm{S}$ & von Braun et al. (2011) \\
\hline HAT-P-21 & 10.2 & $4 \mathrm{~J}$ & Haywood (2008) \\
\hline HD109749 & 10.3 & $\sim 0.3 \mathrm{~J}=\mathrm{S}$ & Haywood (2008) \\
\hline Kepler-10 & 10.6 & $\mathrm{~b}=3.3 \mathrm{~J}, \mathrm{c}=17 \mathrm{~J}$ & Haywood (2008) \\
\hline CoRoT-17 & 10.7 & hot $2.4 \mathrm{~J}$ & Haywood (2008) \\
\hline CoRoT-24 & 11 & $\mathrm{~b}, \mathrm{c}=\mathrm{N}, \mathrm{N}$ & Haywood (2008) \\
\hline WASP-37 & 11 & $1.8 \mathrm{~J}$ & Haywood (2008) \\
\hline WASP-6 & 11 & $0.88 \mathrm{~J}$ & Haywood (2008) \\
\hline WASP-11-HAT-P-10 & 11.2 & $0.8 \mathrm{~J}$ & Haywood (2008) \\
\hline WASP-19 & 11.5 & $\sim 1.1 \mathrm{~J}$ & Haywood (2008) \\
\hline WASP-97 & 11.9 & hot $1.3 \mathrm{~J}$ & Haywood (2008) \\
\hline HD152581 & 12 & $1.5 \mathrm{~J}$ & Haywood (2008) \\
\hline HD190360 = G1777a & 12.11 & $0.06 \mathrm{~J}=\mathrm{N}, \sim 1.6 \mathrm{~J}$ & Haywood (2008) \\
\hline HD99109 & 12.2 & $0.5 \mathrm{~J}$ & Haywood (2008) \\
\hline HAT-P-18 & 12.4 & $0.1 \mathrm{~J}=\mathrm{S}$ & Haywood (2008) \\
\hline HAT-P-22 & 12.4 & $2 \mathrm{~J}$ & Haywood (2008) \\
\hline PSR1719-14 & 12.5 & $\sim 1 \mathrm{~J}$ & Haywood (2008) \\
\hline HD164922 & 13.4 & $\sim 0.4 \mathrm{~J}=\mathrm{S}$ & Haywood (2008) \\
\hline WASP-29 & 15 & $0.25 \mathrm{~J}=\mathrm{S}$ & Haywood (2008) \\
\hline rho Indus & 12.959 & $2 \mathrm{~J}$ & exoplanet.eu + exoplanets.org \\
\hline $\mathrm{SAO} 38269=\mathrm{BD}=48738$ & 12.217 & $\sim 1 \mathrm{~J}$ & Haywood (2008) \\
\hline OGLE2005-BLG-071L & 11.404 & $3 \mathrm{~J}$ & Haywood (2008) \\
\hline DP Leonis & 11.23 & $6 \mathrm{~J}$ & Haywood (2008) \\
\hline HD37605 & 10.712 & $\mathrm{~b}, \mathrm{c}=\mathrm{J}, \mathrm{J}$ & Haywood (2008) \\
\hline OGLE2003-BLG-235L & 10.471 & $2 \mathrm{~J}$ & Haywood (2008) \\
\hline MOA2007-BLG-192L & 10.42 & $3 \mathrm{E}=\mathrm{sE}$ & Haywood (2008) \\
\hline MOA2009-BLG-387L & 10.266 & $\sim 3 \mathrm{~J}$ & Haywood (2008) \\
\hline NN Serpent's & 10.153 & $\mathrm{~b}=7 \mathrm{~J}, \mathrm{c}=2 \mathrm{~J}$ & Haywood (2008) \\
\hline iota $\operatorname{Draco}=12$ Dra & 10.015 & $12 \mathrm{~J}$ & Haywood (2008) \\
\hline
\end{tabular}


Table A.1. (Cont.)

\begin{tabular}{llll}
\hline Star name & Age $(\mathrm{Gyr})$ & Planets & Refs, notes \\
\hline Gliese 649 & 9.998 & $1 \mathrm{~S}$ & Haywood (2008) \\
18 Delphini & 9.897 & $10 \mathrm{~J}$ & Haywood (2008) \\
OGLE2005-BLG-390L & 9.587 & $5.5 \mathrm{E}=\mathrm{sE}$ & Haywood (2008) \\
WASP-5 & 9.582 & $1.6 \mathrm{~J}$ & Haywood (2008) \\
Gliese 253 & 9.451 & $\mathrm{~b}, \mathrm{c}=\mathrm{N}, \mathrm{N}$ & Haywood (2008) \\
HD1690 & 9.332 & 6J & Haywood (2008) \\
WASP-33 = HD15082 & 9.106 & $\sim 4 \mathrm{~J}$ & Haywood (2008) \\
WASP-23 & 9.033 & $\sim 1 \mathrm{~J}$ & Haywood (2008) \\
OGLE2005-BLG-169L & 9.623 & $1 \mathrm{U}$ & Haywood (2008) \\
Kepler-108 = KOI119.02 & $8.9 \pm 3.7$ & $8 \mathrm{E}$ & NASA Exoplanet archive \\
Kepler-444 & $11.23 \pm 0.99$ & $\mathrm{~b}, \mathrm{c}, \mathrm{d}, \mathrm{e}, \mathrm{f}-$ all < Venus & Campante et al. (2015), astroseismology \\
\hline
\end{tabular}

Where possible, the mass of the planet is given, where the following abbreviations are used: J, Jupiter mass; S, Saturnian mass; N, Neptunian mass; E, Earth mass; sE, super-Earth. Along with the reference for the age, the method of determination is given, where possible.

\section{Appendix B. The 'Near 100' - a subset from the nearest 100 star systems.}

Table A.2. Selection from the 'Near 100' of the stars with estimated ages

\begin{tabular}{|c|c|c|c|}
\hline Star & Age (Gyr) & Planet(s) & Notes on Planets \\
\hline GJ $338 \mathrm{AB}$ & $0.025-0.3$ & No & \\
\hline GJ 1 & $(0.1 \pm 0.1) \times 10^{-3}$ & No & \\
\hline GJ 406 & $0.1-0.35$ & No & \\
\hline GJ 873 & $0.1-0.9$ & No & \\
\hline GJ $876 A$ & $0.1-5.0$ & Yes, 4 & $b=2.2756 \pm 0.0045 \mathrm{~J}, \mathrm{c}=0.714 \mathrm{~J}, \mathrm{~d}=0.0215 \mathrm{~J}, \mathrm{e}=0.046 \mathrm{~J} ; 2$ in $\mathrm{HZ}$ \\
\hline GJ 1111 & 0.2 & No & \\
\hline GJ 244A & $0.2-0.3$ & No & \\
\hline GJ 244B & $0.2-0.3$ & No & \\
\hline GJ 144 & $0.2-0.8$ & Yes, 1 & $(1.55 \pm 0.24) \mathrm{J}$ \\
\hline GJ 566A & 0.2 & No & \\
\hline GJ $65 \mathrm{~A}$ & $<1$ & No & \\
\hline GJ 65B & $<1$ & No & \\
\hline GJ 729 & $<1$ & No & \\
\hline GJ 768 & $<1$ & No & \\
\hline GJ 881 & $0.4 \pm 0.04$ & Yes, 1 & $<2-3 \mathrm{~J}$ \\
\hline GJ 674 & $0.55 \pm 0.45$ & Yes, 1 & $\geq 11.8 \mathrm{E}$ \\
\hline GJ 176 & 0.56 & Yes, 1 & $>8.4 \mathrm{E}$ \\
\hline GJ $663 \mathrm{~A}$ & $0.6-1.8$ & No & \\
\hline GJ 280B & 1.37 & No & \\
\hline GJ 440 & 1.44 & No & \\
\hline GJ 702A & 1.9 & No & \\
\hline GJ $667 \mathrm{C}$ & $2-10,2$ & Yes, 2 & $\mathrm{~b} \geq 5.661 \pm 0.437 \mathrm{E}, \mathrm{c} \geq 3.709 \pm 0.682 \mathrm{E} ; 1$ in $\mathrm{HZ}$ \\
\hline GJ 876 & $2.5 \pm 2.4$ pop. I & Yes, 4 & $\mathrm{~b}=2.2756 \pm 0.0045 \mathrm{~J}, \mathrm{c}=0.7142 \pm 0.0039 \mathrm{~J}, \mathrm{~d}=6.83 \pm 0.4 \mathrm{E}, \mathrm{e}=14.6 \pm 1.7 \mathrm{E}$ \\
\hline GJ $280 \mathrm{~A}$ & 3 & No & \\
\hline GJ 849 & $>3$ middle age dwarf & Yes, 2 & $\mathrm{~b}=0.90 \pm 0.04 \mathrm{~J}, \mathrm{c}=0.77 \mathrm{~J}$ \\
\hline GJ 35 & 4 & No & \\
\hline GJ 764 & 4.7 & No & \\
\hline GJ 551 & 4.85 & No & \\
\hline GJ 442A & $4.5-5.7$ & Yes, 1 & $0.05 \pm 0.008 \mathrm{~J}$ \\
\hline GJ 71 & 5.8 & Yes, 5 & $\mathrm{~b}=2.0 \pm 0.8 \mathrm{E}, \mathrm{c}=3.1 \pm 1.4 \mathrm{E}, \mathrm{d}=3.6 \pm 1.7 \mathrm{E}, \mathrm{e}=4.3 \pm 2.01 \mathrm{E}, \mathrm{f}=0.783 \pm 0.012 \mathrm{E}$ \\
\hline GJ 411 & $5.0-10.0$ & No & \\
\hline GJ 559A & $5.0-7.0$ & No & \\
\hline GJ 559B & $5.0-7.0$ & Yes, 1 & 1.13E, Outside HZ \\
\hline GJ 34A & $5.4 \pm 0.9$ & No & \\
\hline GJ 1221 & 5.69 & No & \\
\hline GJ $820 \mathrm{~A}$ & $6.1 \pm 1$ & No & \\
\hline GJ 820B & $6.1 \pm 1$ & No & \\
\hline GJ 139 & $6.1-12.7$ & Yes, $3 \mathrm{sE}$ & $\mathrm{b} \geq 2.7 \pm 0.3 \mathrm{E}, \mathrm{c} \geq 2.4 \pm 0.4, \mathrm{~d} \geq 4.8 \pm 0.6 \mathrm{E}$ \\
\hline GJ 506 & $6.1-6.6$ & Yes, 3 & $\mathrm{~b}=5.3 \pm 0.5 \mathrm{E}, \mathrm{c}=18.8 \pm 1.1 \mathrm{E}, \mathrm{d}=23.7 \pm 2.7 \mathrm{E}$ \\
\hline GJ 380 & 6.6 & No & \\
\hline GJ 780 & $6.6-6.9$ & No & Best SETI Target acc. To Turnbull \& Tarter $(2003 a, b)$ \\
\hline GJ 223.2 & $6.82 \pm 0.02$ & No & \\
\hline
\end{tabular}




\begin{tabular}{llll}
\hline Star & Age $(\mathrm{Gyr})$ & Planet(s) & Notes on Planets \\
\hline GJ 785 & $7.5-8.9$ & Yes, & $\mathrm{b} \geq 16.9 \pm 0.9 \mathrm{E}, \mathrm{c} \geq 24 \pm 5 \mathrm{E}$ \\
GJ 783A & 7.7 & No & \\
GJ 581 & $8 \pm 1$ & Yes, 3 & $\mathrm{e}>1.7 \pm 0.2 \mathrm{E}, \mathrm{b}>15.8 \pm 0.3 \mathrm{E}, \mathrm{c}>5.5 \pm 0.3 \mathrm{E}$; 2 in HZ \\
GJ 832 & 9.24 & Yes, 2 & $\mathrm{~b} \geq 0.64 \pm 0.06) \mathrm{J}, \mathrm{c} \geq 5.4 \pm 1 \mathrm{E}$ \\
GJ 191 & 10 & Yes, & $(4.8 \pm 1) \mathrm{E}$ \\
GJ 699 & $\sim 10$ & No & \\
GJ 892 & 12.46 & No & \\
\hline
\end{tabular}

Information on planetary system is also of importance in devising future space missions focusing on astrobiology. Thus, a column is included in the table giving information on whether a particular star has a planet or not, and planetary masses are given in the last column.

Note: Some of the catalogues used in the study: The Open Exoplanet Catalogue; Extrasolar Planets Encyclopaedia; NASA Exoplanet Archive; Exoplanets Data Explorer. 